The Open Microbiology Journal
CrossMark
Dont: $10.2174 / 1874285801610010202$

RESEARCH ARTICLE

\title{
Matrix-assisted Laser Desorption Ionization-Time-of-Flight Mass Spectrometry (MALDI-TOF MS) as a Reliable Tool to Identify Species of Catalase-negative Gram-positive Cocci not Belonging to the Streptococcus Genus
}

\author{
Marisa Almuzara ${ }^{1, *}$, Claudia Barberis ${ }^{1}$, Viviana Rojas Velázquez ${ }^{1}$, Maria Soledad Ramirez ${ }^{2}$, Angela \\ Famiglietti $^{1}$ and Carlos Vay ${ }^{1}$ \\ ${ }^{\prime}$ Universidad de Buenos Aires, Facultad de Farmacia y Bioquímica, Laboratorio de Bacteriología, Departamento de \\ Bioquímica Clínica Hospital de Clínicas José de San Martín Ciudad Autónoma de Buenos Aires, Argentina \\ ${ }^{2}$ Center for Applied Biotechnology Studies, Department of Biological Science, California State University Fullerton, \\ Fullerton, CA, USA
}

Received: March 05, 2016

Revised: November 04, 2016

Accepted: November 11, 2016

Abstract:

Objective:

To evaluate the performance of matrix-assisted laser desorption ionization-time-of-flight mass spectrometry (MALDI-TOF MS) by using 190 Catalase-negative Gram-Positive Cocci (GPC) clinical isolates.

\section{Methods:}

All isolates were identified by conventional phenotypic tests following the proposed scheme by Ruoff and Christensen and MALDITOF MS (Bruker Daltonics, BD, Bremen, Germany). Two different extraction methods (direct transfer formic acid method on spot and ethanol formic acid extraction method) and different cut-offs for genus/specie level identification were used. The score cut-offs recommended by the manufacturer $(\geq 2.000$ for species-level, 1.700 to 1.999 for genus level and $<1.700$ no reliable identification) and lower cut-off scores $(\geq 1.500$ for genus level, $\geq 1.700$ for species-level and score $<1.500$ no reliable identification) were considered for identification. A minimum difference of $10 \%$ between the top and next closest score was required for a different genus or species.

MALDI-TOF MS identification was considered correct when the result obtained from MS database agreed with the phenotypic identification result.

When both methods gave discordant results, the $16 \mathrm{~S}$ rDNA or sodA genes sequencing was considered as the gold standard identification method. The results obtained by MS concordant with genes sequencing, although discordant with conventional phenotyping, were considered correct. MS results discordant with 16S or sodA identification were considered incorrect.

\section{Results:}

Using the score cut-offs recommended by the manufacturer, $97.37 \%$ and $81.05 \%$ were correctly identified to genus and species level, respectively. On the other hand, using lower cut-off scores for identification, $97.89 \%$ and $94.21 \%$ isolates were correctly identified to genus and species level respectively by MALDI-TOF MS and no significant differences between the results obtained with two extraction methods were obtained.

\footnotetext{
* Address correspondence to this author at the Universidad de Buenos Aires, Facultad de Farmacia y Bioquímica, Laboratorio de Bacteriología, Departamento de Bioquímica Clínica Hospital de Clínicas José de San Martín Ciudad Autónoma de Buenos Aires, Argentina; Tel: +54 11 59508663; Fax: +54 11 59508691; E-mail: marisaalmuzara@gmail.com
} 


\section{Conclusion:}

The results obtained suggest that MALDI-TOF MS has the potential of being an accurate tool for Catalase-negative GPC identification even for those species with difficult diagnosis as Helcococcus, Abiotrophia, Granulicatella, among others. Nevertheless, expansion of the library, especially including more strains with different spectra on the same species might overcome potential "intraspecies" variability problems. Moreover, a decrease of the identification scores for species and genus-level identification must be considered since it may improve the MALDI-TOF MS accuracy.

Keywords: Catalase-negative gram-positive Cocci, Enterococcus spp, Identification, MALDI-TOF MS.

\section{INTRODUCTION}

The Catalase-negative Gram-positive cocci (GPC) are among the microorganisms that are isolated more frequently in the microbiology laboratory. In most clinical laboratories, their identification is currently performed by conventional microbiological methods and, in some cases, by molecular methods as 16S rDNA gene and other housekeeping genes sequencing [1]. Although both methodologies achieve a reliable identification, these are time consuming and laborintensive.

The correct identification of catalase negative GPC has clinical impact since for e.g. misidentification of certain species of Enterococcus (E. gallinarum or E. casseliflavus) could lead to failure of treatment if vancomycin is used. Likewise, misidentification of Abiotrophia/Granulicatella as Streptococcus viridans group might have a negative impact if low doses of penicillin are used for treatment.

The availability of robust diagnostic tools for catalase negative GPC identification has allowed to establish the role of several bacteria originally considered nonpathogenic for humans as responsible for infections in immunocompromised or debilitated patients. For example, Lactococcus and Vagococcus were considered for a long time. Enterococcus variants [2], only through the use of new identification techniques have been recognized as new human pathogens.

Recently, many studies have evaluated the performance of MALDI-TOF MS for bacterial identification [3 - 8]. In this sense, correct identification for Enterococcus spp. by mass spectrometry at the species level has been reported by other authors [9, 10]. However, few works regarding Catalase-negative GPC identification by MALDI-TOF MS including a limited number of species $[11,12]$ or using an earlier database $[11,13]$ have been published.

\section{MATERIALS AND METHODS}

\section{Bacterial Isolates}

A collection of one hundred and ninety Catalase-negative GPC patients' clinical isolates that were recovered between 1998 and 2014 at the Hospital de Clínicas José de San Martín, Universidad de Buenos Aires, Argentina, were analyzed by MS by the Bruker Daltonics MicroFlex LT instrument using MALDI Biotyper software 3.1 (Bruker Daltonics, Bremen, Germany). This study included 96 Enterococcus spp. isolates and 94 isolates of catalase-negative GPC (excluding Streptococcus spp.) as Leuconostoc; Aerococcus; Granulicatella/Abiotrophia; Globicatella; Helcococcus; Lactococcus; Gemella; Pediococcus; Facklamia, Vagococcus; Weisella). All isolates had clinical relevance and were isolated from the following samples: $70 \%$ from respiratory specimens, $10 \%$ from blood, $10 \%$ from surgical samples, 5\% from urine and 5\% from other clinical sources.

All isolates, previously preserved at $-70^{\circ} \mathrm{C}$ in stock medium, were subcultured twice consecutively on $5 \%$ sheep blood agar and incubated for $24-48 \mathrm{~h}$ in a $5 \% \mathrm{CO}_{2}$ atmosphere at $35^{\circ} \mathrm{C}$, prior to study.

\section{Phenotypic and Molecular Identification}

The standard biochemical identification was carried out following the proposed scheme by Ruoff and Christensen [14 - 16]. First, colony morphology on sheep blood agar plates and its microscopic morphology on the Gram stain were observed. The determination of physiological tests, such as: production of pyrrolidonyl arylamidase (PYR), production of leucineaminopeptidase (LAP), growth in broth containing $6.5 \% \mathrm{NaCl}$, vancomycin susceptibility; hemolysis on Trypticase soy agar with 5\% sheep blood, motility, hydrolysis bile esculin, hippurate hydrolysis, and satellitism, test for growth at $10^{\circ}$ and $45^{\circ} \mathrm{C}$, among others was also performed. In addition, 16S rDNA gene or sodA (coding for the manganese-dependent superoxide dismutase) sequencing were also used in those species for which it is difficult to reach definitive identification by using conventional biochemical tests or to solve discrepancies between conventional 
methodology and MALDI-TOF MS identification. Using the universal primers described by Weisburg et al. [17] and following the manufacturer's specifications for the Taq DNA polymerase (Promega), 16S rDNA PCR products were obtained. In the case of $\operatorname{sod} A$ gene, the methodology described by Poyart et al. [18] was used. Sequencing of the PCR products was performed on both DNA strands using ABIPrism 3100 BioAnalyzer equipment at Macrogen Inc. sequencing facility, South Korea. The sequences were analyzed by BLAST analysis using two databases (GenBank and EMBL-Bank) to perform the sequence comparison.

\section{Sample Preparation for MALDI-TOF MS}

Two methods: i) direct transfer formic acid method on spot and ii) ethanol formic acid extraction have been performed to prepare bacteria for identification by MALDI-TOF MS. For the direct transfer-formic acid method, MALDI-TOF target plates were inoculated into the spots by picking a freshly grown overnight colony and overlaid with $1 \mu \mathrm{l}$ of $70 \%$ formic acid (Sigma-Aldrich). Each spot was allowed to dry and subsequently overlaid with $1 \mu \mathrm{l}$ of matrix solution (a-cyano-4hydroxycinnamic acid in 50\% acetonitrile, 2.5\% TFA). For the ethanol formic extraction method, a loopful of bacteria was transferred directly into a tube with $300 \mu \mathrm{l}$ of distilled water and mixed thoroughly to suspend the cell effectively in the water. and $900 \mu \mathrm{l}$ etanol (100\%) was added later. The cell suspension was centrifuged at 13,000 rpm for $2 \mathrm{~min}$, and the supernatant was decanted. After a second centrifugation in the same conditions as previously specified, residual ethanol was removed from the pellet using a pipet. The pellet was air dried and thoroughly resuspended in $50 \mu 170 \%$ formic acid and an equal volume of acetonitrile. After centrifugation at 13,000 $\mathrm{rpm}$ for $2 \mathrm{~min}, 1 \mu \mathrm{l}$ of the supernatant was transferred to the MALDI TOF target plate and allowed to dry at room temperature before being overlaid with $1 \mu \mathrm{l}$ of HCCA matrix solution [12].

\section{MALDI-TOF MS Analysis}

Mass spectra were acquired using the MALDI-TOF MS spectrometer in a linear positive mode (Microflex LT mass spectrometer, Bruker Daltonics, Germany). Biotyper library version 3.0 and MALDI Biotyper software version 3.1 were used for bacterial identification with default parameters setting (positive linear mode; laser frequency $60 \mathrm{~Hz}$; ion source 1 voltage, $20 \mathrm{kV}$; ion source 2 voltage, $16.7 \mathrm{kV}$; lens voltage, $7.0 \mathrm{kV}$; mass range, 2,000 to 20,000 Da). For each spectrum, 240 laser shots in 40-shot steps from different areas of the sample spot were accumulated and analyzed (automatic mode, default setting). The Bruker Bacterial Test Standard (BTS) (Bruker Daltonics, Germany), an extract of Escherichia coli supplemented with RNase A and myoglobin, was used for instrument calibration according to the instructions of the manufacturer. Each specimen was run in duplicate.

With the purpose of improving the available database for Globicatella, due to the fact that in the MALDI Biotyper database, G. sanguinis is not included, the MALDI-TOF MS analysis of the spectra obtained for eigth G. sanguinis clinical isolates was performed. The 24 spectra obtained from the eight spots for each G. sanguinis strains were analyzed by FlexAnalysis (version 3.0, Bruker Daltonics). Finally, a minimum of 20 accurate spectra were downloaded in MALDI Biotyper sofware (version 3.0, Bruker Daltonics) to create a main spectrum profile (MSP) of each strain according to the manufacturer's recommendations.

\section{MALDI-TOF MS Data Interpretation}

Different cut-offs for genus/specie level identification were used in order to depict the identification results. The score cut-offs recommended by the manufacturer were used to determine species-level identification $\geq 2.000$, genus level identification, 1.700 to 1.999 and $<1.700$ unreliable identification. Additionally, based on previous studies [4, 7, 19] and on our own results [20], lower cut-off scores for identification were used as following: $\geq 1.500$ for genus level, $\geq$ 1.700 for species-level and score $<1.500$ was considered as no reliable identification. Moreover, a $10 \%$ difference between the first two diagnostic species having the best matches in the database was required to give species identification. If these conditions were not met, identification was considered correct only at the genus level [19].

MALDI-TOF identification was considered correct when the result obtained from MS database agreed with the phenotypic identification result.

When both methods gave discordant results, we carried out the $16 \mathrm{~S}$ rDNA or sodA sequencing as the gold standard identification method. The results obtained by MS concordant with identification by $16 \mathrm{~S}$ rDNA or $\operatorname{sodA}$ sequencing were considered correct. MS results discordant with 16S rDNA or sodA identification were considered incorrect. 


\section{Statistical Analysis}

Population parameters of both extraction methods were compared using the $\mathrm{z}$ test [21].

\section{RESULTS AND DISCUSSION}

Using the direct transfer formic acid method and lower cut-off scores for identification, 186 isolates $(97.89 \%)$ to the genus level and 179 isolates (94.21\%) to the species level were correctly identified by MALDI-TOF MS (Tables 1 and 2). On the other hand, the results obtained using the ethanol formic acid-acetonitrile extraction methods were: correct identification of 185 isolates $(97.3 \%)$ to the genus level and 177 isolates $(93.1 \%)$ to the species level. These results clearly showed no significant differences between the results obtained with both the methods of extraction (with confidence levels of $95 \%$ and $99 \%$ ).

Table 1. Agreement of MALDI-TOF MS with standard biochemical or molecular identifications for Enterococcus spp. using different identification cutoff scores.

\begin{tabular}{|c|c|c|c|c|c|c|c|}
\hline $\begin{array}{l}\text { Conventional or molecular } \\
\text { identification method }\end{array}$ & No of isolates & Genus ID (score $>1.5$ ) & Species ID $($ score $>1.7)$ & No ID & $\begin{array}{c}\text { Genus ID } \\
(\text { score }>1.7)\end{array}$ & $\begin{array}{c}\text { Species ID } \\
(\text { score }>\mathbf{2 . 0})\end{array}$ & No ID \\
\hline Enterococcus faecalis & 20 & 20 & 20 & & 20 & 20 & \\
\hline Enterococcus faecium & 20 & 20 & 20 & & 20 & 20 & \\
\hline Enterococcus raffinosus & 11 & 11 & 11 & & 11 & 9 & \\
\hline Enterococcus gallinarum & 7 & 7 & 7 & & 7 & 7 & \\
\hline Enterococcus casseliflavus & 11 & 11 & 11 & & 11 & 10 & \\
\hline Enterococcus avium & 11 & 11 & 11 & & 11 & 11 & \\
\hline Enterococcus hirae & 7 & 7 & 7 & & 7 & 7 & \\
\hline Enterococcus mundtii & 2 & 2 & 2 & & 2 & 2 & \\
\hline Enterococcus devriesei & 1 & 1 & 1 & & 1 & 1 & \\
\hline Enterococcus durans & 5 & 5 & 5 & & 5 & 5 & \\
\hline Enterococcus malodoratus & 1 & 1 & 1 & & 1 & 1 & \\
\hline SubTotal n (\%) & 96 & $96(100 \%)$ & $96(100 \%)$ & $0(0 \%)$ & $96(100 \%)$ & $93(95.83 \%)$ & $0(0 \%)$ \\
\hline
\end{tabular}

Table 2. Agreement of MALDI-TOF MS with standard biochemical or molecular identifications for related genera using different identification cutoff scores.

\begin{tabular}{|c|c|c|c|c|c|c|c|}
\hline 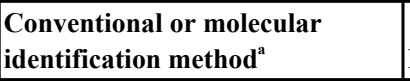 & No of isolates & $\begin{array}{c}\text { Genus ID }^{\mathrm{b}} \text { (score } \\
>1.5)\end{array}$ & $\begin{array}{c}\text { Species ID (score } \\
>1.7)\end{array}$ & NO ID ${ }^{c}$ & $\begin{array}{c}\text { Genus ID } \\
(\text { score }>1.7)\end{array}$ & $\begin{array}{l}\text { Species ID } \\
(\text { score }>\mathbf{2 . 0})\end{array}$ & NO ID \\
\hline Leuconostoc mesenteroides & 8 & 8 & 8 & & 8 & 7 & \\
\hline Leuconostoc pseudomesenteroides & 3 & 3 & 3 & & 3 & 1 & \\
\hline Leuconostoc lactis & 4 & 4 & 4 & & 4 & 3 & \\
\hline Aerococcus viridans & 11 & 9 & 9 & 2 & 8 & 1 & 2 \\
\hline Aerococcus urinae & 5 & 5 & 5 & & 5 & 5 & \\
\hline Abiotrophia defectiva & 5 & 5 & 5 & & 5 & 2 & \\
\hline Granulicatella adiacens & 10 & 10 & 9 & & 9 & 9 & 1 \\
\hline Granulicatella elegans & 2 & 1 & 1 & 1 & 1 & 1 & 1 \\
\hline Globicatella sanguinis & 5 & 5 & 5 & & 5 & 3 & \\
\hline Helcococcus kunzii & 7 & 7 & 7 & & 7 & 7 & \\
\hline Lactococcus lactis & 9 & 9 & 9 & & 9 & 9 & \\
\hline Lactococcus garviae & 1 & 1 & 1 & & 1 & 0 & \\
\hline Gemella morbillorum & 3 & 3 & 3 & & 3 & 3 & \\
\hline Gemella haemolysans & 5 & 4 & 4 & 1 & 4 & 3 & 1 \\
\hline Gemella sanguinis & 2 & 2 & 2 & & 2 & 2 & \\
\hline Pediococcus acidilactici & 1 & 1 & 1 & & 1 & 1 & \\
\hline Pediococcus pentosaceus & 4 & 4 & 4 & & 4 & 2 & \\
\hline Facklamia languida & 1 & 1 & 1 & & 1 & & \\
\hline Facklamia hominis & 1 & 1 & 1 & & 1 & 1 & \\
\hline Vagococcus sp. & 6 & 6 & 0 & & 6 & 0 & \\
\hline Weissella viridescens & 1 & 1 & 1 & & 1 & 1 & \\
\hline
\end{tabular}


(Table $\square$ ) contd.....

\begin{tabular}{|c|c|c|c|c|c|c|c|}
\hline 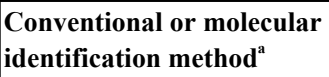 & No of isolates & $\begin{array}{c}\text { Genus ID }^{b} \text { (score } \\
>1.5)\end{array}$ & $\begin{array}{c}\text { Species ID (score } \\
>1.7)\end{array}$ & NO ID ${ }^{c}$ & $\begin{array}{c}\text { Genus ID } \\
(\text { score }>1.7)\end{array}$ & \begin{tabular}{|c|} 
Species ID \\
$($ score $>\mathbf{2 . 0})$
\end{tabular} & NO ID \\
\hline SubTotal n (\%) & 94 & $90(95.75 \%)$ & $83(88.29 \%)$ & $4(4.25 \%)$ & $89(94.6 \%)$ & $61(64.89 \%)$ & $5(5.32 \%)$ \\
\hline Total n (\%) & 190 & $186(97.89 \%)$ & $179(94.21 \%)$ & $4(2.10 \%)$ & $185(97.37 \%)$ & $154(81.05 \%)$ & $5(2.63 \%)$ \\
\hline
\end{tabular}

${ }^{\mathrm{a}}$ Table format adapted from reference [3]; ${ }^{\mathrm{b}}$ ID: identification; ${ }^{\mathrm{c}} \mathrm{NO}$ ID: not reliable identification

Using a lower score $(\geq 1.7)$ for the species-level identification when analyzing the included Enterococcus isolates, the species-level identification increased from 93 Enterococcus isolates $(95.83 \%)$ to 96 isolates $(100 \%)$ respectively. Moreover, in the case of related genera, the use of lower scores showed a significant increase in the identification rate to species level from $64.89 \%$ (61 isolates) to $88.29 \%$ ( 83 isolates). No misidentification results were observed from changing the genus or species score cut-offs (Tables $\mathbf{1}$ and $\mathbf{2}$ ).

In accordance with Schulthess et al. [12], the Bruker MALDI Biotyper system using the direct transfer-formic acid sample preparation method was shown to be a highly reliable tool for the identification of GPC. Furthermore, in agreement with our results, these authors found no significant difference between the two extraction methods used for sample preparation.

Here, we included 94 isolates of catalase-negative GPC (excluding Streptococcus spp.). In a previous work, Alatoom et al. [11] included 48 isolates of related genera to Enterococcus and Streptococcus. However, species as Leuconostoc mesenteroides, Leuconostoc pseudomesenteroides, Leuconostoc lactis, Gemella haemolysans, Gemella sanguinis, Gobicatella sanguinis, Vagococcus spp., among others, were not included in previous works. Similarly, Schulthess B et al. study [12] included 33 isolates of related genera with lower diversity of species: Aerococcus viridans and a few other genera as Abiotrophia, Gemella, Granulicatella, Lactococcus and Leuconostoc.

MALDI-TOF MS had an excellent performance in identifying all Enterococcus species including those of uncommon isolation and difficult identification by conventional biochemical tests as Enterococcus malodoratus and Enterococcus devriesei and also those species most biochemically inert such as Enterococcus hirae and Enterococcus durans. Our results are in agreement with those published by other authors $[9,10]$ showing the reliability of MALDITOF MS in the identification of Enterococcus species.

Regarding Aerococcus spp., using the cut-off scores recommended by the manufacturer, only 1 of the 11 Aerococcus viridans isolates was identified to the species level. On the other hand, all Aerococcus urinae isolates tested were correctly identified to species level, regardless of the score cut-offs used.

Helcococcus kunzii, a fastidious species is difficult to identify due to its phenotypic similarity to A. viridans which shares the morphology in liquid medium, sensitivity to vancomycin, production of pyrrolidonyl arylamidase and growth in broth $\mathrm{NaCl} 6.5 \%$. Esculin hydrolysis was correctly identified at species level by MALDI-TOF MS with both of the extraction procedures used. Alatoom et al. [11] also reported a correct identification of $H$. kunzii to the species level when they used the protein extraction method. In contrast, when they used the direct colony method, the H. kunzii isolates were only identified to genus level [11].

When Lactococcus spp. was tested, all L. lactis isolates were correctly identified to species level with high scores (2.116 to 2.511) opposed to only one $L$. garviae isolate, which was identified with a mean score of 1.849 .

With a single Gemella isolate (G. haemolysans), a reliable identification was not obtained; the remaining Gemella isolates were identified to the species level.

MALDI-TOF MS exhibited a reliable performance in identifying Globicatella sanguinis (all isolates were identified to the species level). Since the only species that is included in the Bruker database is Globicatella sulfidifaciens, this result was successfully achieved due to the previous incorporation of 8 clinical spectra of G. sanguinis strains in the database.

The identification of Vagococcus spp. isolates tested by MALDI-TOF MS as V. fluvialis could not be reliably confirmed since the correct identification at the species level of this genus cannot be achieved either by the traditional methods of identification or by the $16 \mathrm{~S}$ rDNA gene or the $\operatorname{sod} A$ gene sequencing. In addition, the Bruker database only contained 4 spectra of $V$. fluvialis; the spectra of other species were not included. The sodA sequence analysis of the six Vagococcus isolates tested revealed only $81-83 \%$ of sequence identity with Vagococcus salmoninarum. These results indicate that in this particular case, the identification is reliable only to genus level. The exposed results could be explained in part due to the small number of Vagococcus' sodA sequences available in the databases.

MALDI-TOF MS allows us to identify some L. lactis isolates with an unusual biochemical profile (positive 
arabinose fermentation), being misidentified by conventional identification schemes as Enterococcus faecium. Moreover, an Aerococcus viridans isolate that hydrolyzed arginine and therefore was erroneously identified as Aerococcus sanguinicola by standard biochemical tests was solved by MALDI-TOF. Further testing of these isolates by 16S rDNA gene sequencing confirmed that the Biotyper identification was correct.

In conclusion, our results showed that the extraction preparation method would not be necessary since it did not expose a significant increase in the percentage of identification when was compared with the direct transfer formic acid method. Furthermore, MALDI- TOF MS allows us to recognize isolates with atypical biochemical as L. lactis or A.viridans. All the results exposed above suggest that MALDI-TOF MS has the potential of being an accurate tool for catalase negative GPC identification even for species with difficult diagnosis as Helcococcus, Abiotrophia, Granulicatella, among others. Nevertheless, expansion of the library, especially including more strains with similar spectra and a decrease of the identification scores for species- and genus-level identifications may improve MALDITOF MS accuracy.

\section{CONFLICT OF INTEREST}

The authors confirm that this article content has no conflict of interest.

\section{ACKNOWLEDGEMENTS}

This work was supported by grants from the "Secretaría de Ciencia y Técnica de la Universidad de Buenos Aires" (UBACyT) to Carlos Vay.

\section{REFERENCES}

[1] Bosshard PP, Abels S, Altwegg M, Böttger EC, Zbinden R. Comparison of conventional and molecular methods for identification of aerobic catalase-negative gram-positive cocci in the clinical laboratory. J Clin Microbiol 2004; 42(5): 2065-73.

[http://dx.doi.org/10.1128/JCM.42.5.2065-2073.2004] [PMID: 15131171]

[2] Facklam R, Elliott JA. Identification, classification, and clinical relevance of catalase-negative, gram-positive cocci, excluding the streptococci and enterococci. Clin Microbiol Rev 1995; 8(4): 479-95.

[PMID: 8665466]

[3] Almuzara M, Barberis C, Traglia G, Famiglietti A, Ramirez MS, Vay C. Evaluation of matrix-assisted laser desorption ionization-time-offlight mass spectrometry for species identification of nonfermenting Gram-negative bacilli. J Microbiol Methods 2015; 112 : 24-7. [http://dx.doi.org/10.1016/j.mimet.2015.03.004] [PMID: 25765149]

[4] Bizzini A, Durussel C, Bille J, Greub G, Prodhom G. Performance of matrix-assisted laser desorption ionization-time of flight mass spectrometry for identification of bacterial strains routinely isolated in a clinical microbiology laboratory. J Clin Microbiol 2010; 48(5): 1549-54.

[http://dx.doi.org/10.1128/JCM.01794-09] [PMID: 20220166]

[5] van Veen SQ, Claas EC, Kuijper EJ. High-throughput identification of bacteria and yeast by matrix-assisted laser desorption ionization-time of flight mass spectrometry in conventional medical microbiology laboratories. J Clin Microbiol 2010; 48(3): 900-7. [http://dx.doi.org/10.1128/JCM.02071-09] [PMID: 20053859]

[6] Bizzini A, Jaton K, Romo D, Bille J, Prodhom G, Greub G. Matrix-assisted laser desorption ionization-time of flight mass spectrometry as an alternative to $16 \mathrm{~S}$ rRNA gene sequencing for identification of difficult-to-identify bacterial strains. J Clin Microbiol 2011; 49(2): 693-6. [http://dx.doi.org/10.1128/JCM.01463-10] [PMID: 21106794]

[7] Cherkaoui A, Hibbs J, Emonet S, et al. Comparison of two matrix-assisted laser desorption ionization-time of flight mass spectrometry methods with conventional phenotypic identification for routine identification of bacteria to the species level. J Clin Microbiol 2010; 48(4): 1169-75.

[http://dx.doi.org/10.1128/JCM.01881-09] [PMID: 20164271]

[8] Neville SA, Lecordier A, Ziochos H, et al. Utility of matrix-assisted laser desorption ionization-time of flight mass spectrometry following introduction for routine laboratory bacterial identification. J Clin Microbiol 2011; 49(8): 2980-4. [http://dx.doi.org/10.1128/JCM.00431-11] [PMID: 21632894]

[9] Fang H, Ohlsson AK, Ullberg M, Ozenci V. Evaluation of species-specific PCR, Bruker MS, VITEK MS and the VITEK 2 system for the identification of clinical Enterococcus isolates. Eur J Clin Microbiol Infect Dis 2012; 31(11): 3073-7. [http://dx.doi.org/10.1007/s10096-012-1667-x] [PMID: 22706514]

[10] Lee M, Chung HS, Moon HW, Lee SH, Lee K. Comparative evaluation of two matrix-assisted laser desorption ionization time-of-flight mass spectrometry (MALDI-TOF MS) systems, Vitek MS and Microflex LT, for the identification of Gram-positive cocci routinely isolated in clinical microbiology laboratories. J Microbiol Methods 2015; 113: 13-5. [http://dx.doi.org/10.1016/j.mimet.2015.03.020] [PMID: 25818760]

[11] Alatoom AA, Cunningham SA, Ihde SM, Mandrekar J, Patel R. Comparison of direct colony method versus extraction method for identification of gram-positive cocci by use of Bruker Biotyper matrix-assisted laser desorption ionization-time of flight mass spectrometry. $\mathrm{J}$ 
Clin Microbiol 2011; 49(8): 2868-73.

[http://dx.doi.org/10.1128/JCM.00506-11] [PMID: 21613431]

[12] Schulthess B, Brodner K, Bloemberg GV, Zbinden R, Böttger EC, Hombach M. Identification of Gram-positive cocci by use of matrixassisted laser desorption ionization-time of flight mass spectrometry: comparison of different preparation methods and implementation of a practical algorithm for routine diagnostics. J Clin Microbiol 2013; 51(6): 1834-40.

[http://dx.doi.org/10.1128/JCM.02654-12] [PMID: 23554198]

[13] Christensen JJ, Dargis R, Hammer M, Justesen US, Nielsen XC, Kemp M. Matrix-assisted laser desorption ionization-time of flight mass spectrometry analysis of Gram-positive, catalase-negative cocci not belonging to the Streptococcus or Enterococcus genus and benefits of database extension. J Clin Microbiol 2012; 50(5): 1787-91. [http://dx.doi.org/10.1128/JCM.06339-11] [PMID: 22403420]

[14] Christensen J, Ruoff K. General approaches to identification of aerobic gram-positive cocci. In: Diagnostic Strategies And General Topics. $11^{\text {th }}$ ed. Washington, DC: ASM Press 2015. [http://dx.doi.org/10.1128/9781555817381.ch20]

[15] Christensen J, Ruoff K. Aerococcus, Abiotrophia, and other aerobic catalase-negative gram-positive Cocci. In: Jorgensen JH, Pfaller A, Carroll KC, et al., Eds., Manual of Clinical Microbiology. $11^{\text {th }}$ ed. Washington, DC: ASM Press 2015; pp. 422-36.

[16] Martins Teixeira L, Siqueira Carvalho M, Facklam R, Lynn Shewmaker P. Enterococcus. In: Jorgensen JH, Pfaller A, Carroll KC, et al., Eds., Manual of Clinical Microbiology. $11^{\text {th }}$ ed. Washington, DC: ASM Press 2015; pp. 403-21.

[17] Weisburg WG, Barns SM, Pelletier DA, Lane DJ. 16S ribosomal DNA amplification for phylogenetic study. J Bacteriol 1991; 173(2): 697-703. [http://dx.doi.org/10.1128/jb.173.2.697-703.1991] [PMID: 1987160]

[18] Poyart C, Quesne G, Coulon S, Berche P, Trieu-Cuot P. Identification of streptococci to species level by sequencing the gene encoding the manganese-dependent superoxide dismutase. J Clin Microbiol 1998; 36(1): 41-7. [PMID: 9431917]

[19] Alatoom AA, Cazanave CJ, Cunningham SA, Ihde SM, Patel R. Identification of non-diphtheriae corynebacterium by use of matrix-assisted laser desorption ionization-time of flight mass spectrometry. J Clin Microbiol 2012; 50(1): 160-3. [http://dx.doi.org/10.1128/JCM.05889-11] [PMID: 22075579]

[20] Barberis C, Almuzara M, Join-Lambert O, Ramírez M S, Famiglietti A, Vay C. Comparison of the Bruker MALDI-TOF mass spectrometry system and conventional phenotypic methods for identification of Gram-positive rods. PLoS One 2014; 9(9): e106303. [http://dx.doi.org/10.1371/journal.pone.0106303] [PMID: 25184254]

[21] Sprinthall RC, Ed. Basic statistical analysis. $9^{\text {th }}$ ed. Upper Saddle River, NJ: Pearson Education 2011.

(C) Almuzara et al.; Licensee Bentham Open

This is an open access article licensed under the terms of the Creative Commons Attribution-Non-Commercial 4.0 International Public License (CC BY-NC 4.0)(https://creativecommons.org/licenses/by-nc/4.0/legalcode), which permits unrestricted, non-commercial use, distribution and reproduction in any medium, provided the work is properly cited. 